\title{
High Gain Microstrip Active Antenna utilizes as Transmitter and Receiver with Linear and Circular Polarization
}

\author{
Engr. AHMED. S. ALZAHRANI", \\ Master of Electronics and Communications, Department of Electrical and Computer \\ Engineering, King Abdulaziz University, Saudi Arabia ${ }^{1}$ \\ Prof. Adnan. M. AFFANDI \\ Professor, Department of Electrical and Computer Engineering, King Abdulaziz \\ University, Saudi Arabia \\ *Email: asmz11@hotmail.com
}

\begin{abstract}
In this research paper, a high gain microstrip active antenna is operating as transmitter and receiver at the same time with linear and circular polarization operations. This proposed work contains five essential parts such as high gain and broadband applications. Firstly, mainly is dealing with passive patch antennas. These selected passive antennas enjoy both high gain and broadband applications. Part two intends to convert the selected passive patch antennas into an active antenna. Part three intended to improve the performances of the proposed active integrated antennas. Part four enhancement elements (which is known as parasitic elements) are utilized in order to increase the gain of selected antennas. Part five, this proposed active integrated antennas will be converted from linear polarization (LP) into circulation polarization (CP). All the selected antennas will simulate by using models of ADS Agilent.
\end{abstract}

Keywords: Microstrip, Antenna, Receiver, Transmitter, Linear and Circular Polarization. 


\section{Introduction}

Modern technology is taking off at a breakneck speed towards providing the best antennas that provide the best advanced technology solutions to keep pace with continuous manufacturing based on high-gain antennas (Garg et al, 2001). The proposed antenna can design simultaneous transmit and receive (STAR) antennas that can send and receive a signal simultaneously at the same time (Khoshniat et al, 2011). The antenna's intelligent design and integration with active circuits lead to innovative millimeter-wave and microwave application systems and considerable achievements in low power consumption, small profile, compactness, low cost, and multiple applications (Rogier, Declercq, 2010). This technology caused new designs in military and industrial applications such as transceivers, radar communications, sensors, and wireless (Peter T. et al, 2007). The microstrip antenna is one of the most important devices used in RFID applications. Radio Frequency Identification (RFID) is one of the most recent technologies in the field of wireless communications. This advance should provide a very great service in terms of speed, diversity, and ease (T, Itoh, 1997).

This proposed paper's main task is to design a simulated active integrated antenna at microwave band from $2-3 \mathrm{GHz}$ frequency range to get high gain and wide bandwidth for replacing the duplexers circuit with a patch antenna and an active antenna. The patch antenna single and multiple will be selected according to their performance gain and bandwidth. Low noise amplifier one stage and cascade will be discussed theoretical according to of three terminals as an active part. also will use enhancement elements (which are known as parasitic elements) are utilized in order to increase the gain of selected antennas. we will use the ADS simulation program in this research.

\section{Passive Antenna}

In this work, the design of the desired antenna provides two steps: we first present the design of a rectangular patch antenna based on microstrip technology, and the second we will design a rectangular array patch antenna for obtaining a high gain and directivity of the desired antenna. Single Patch Antenna for the first step, we will start by studying the first antenna which is a single patch printed antenna. We will focus on the design based 
on a small size at a resonant frequency of $2.45 \mathrm{GHz}$. Then we go perform a simulation to find internal parameters (S. parameters, bandwidth) and external characteristics (gain, directivity, and radiation pattern). To increase the total gain of the antenna and have bandwidth wider and taking advantage of the functionality of the radiation overlay of several radiating elements in the same direction, we suggest the second step, which is the more important in order to design an array of antennas grouping patches identical to our first patch antenna proposed in the first step.

The antenna structure proposed as, and shown in Figure 1, has the rectangular patch connected with $50 \mathrm{Ohm}$ line feed. The lower plane and the ground plane are composed of a PEC material (Perfect Electric Conductor). The antenna substrate is FR4, which has a relative permittivity $\varepsilon r=4.6$ and a dielectric loss tangent, $\tan \delta=0.01$.

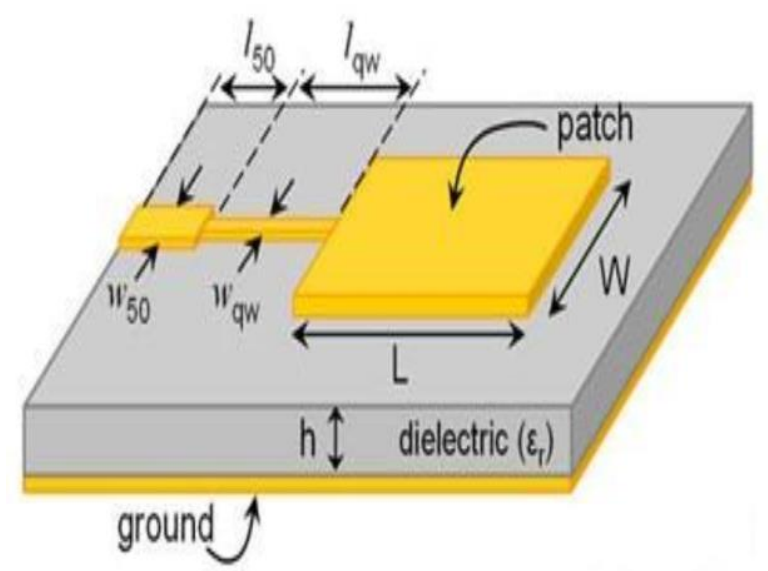

Figure.1 Microstrip Patch Antenna Configuration

For designing a microstrip patch antenna, we have to select the resonant frequency and a dielectric medium for which the antenna is to be designed. The geometry of the proposed patch antenna based on microstrip technology is depicted in figure 2. The dimensions to be calculated are shown in the table.1. 


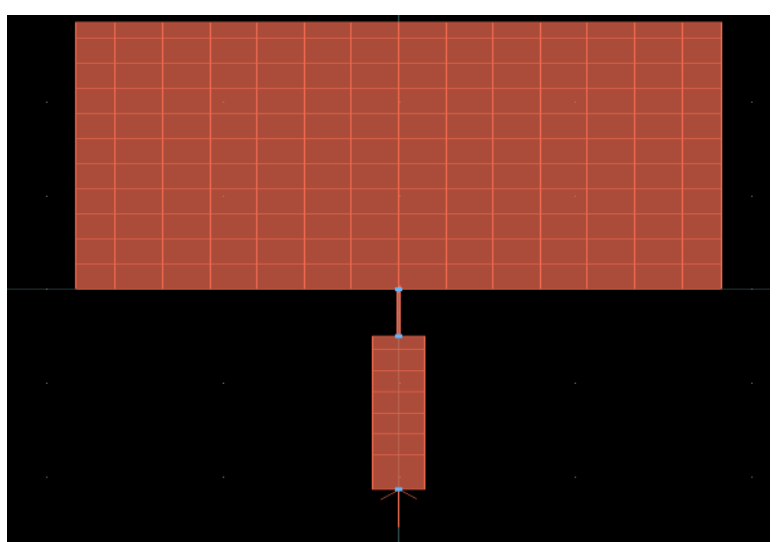

Figure.2 Layout of the single microstrip patch antenna

Table 1: The dimensions of the microstrip patch antenna

\begin{tabular}{|c|c|c|}
\hline Parameters & Description & Value (mm) \\
\hline hs & Thickness of substrate & 1.58 \\
\hline hg & Thickness of ground & 0.035 \\
\hline L & Length of patch & 28.54 \\
\hline W & Width of patch & 36.58 \\
\hline hp & Thickness of patch & 0.035 \\
\hline Lfeed & Length of line feed & 16.43 \\
\hline Wfeed & Width of the line feed & 2.894 \\
\hline La & Length of adaptation & 5 \\
\hline Wa & Width of adaptation & 0.2 \\
\hline
\end{tabular}

\section{a) Array Patch Antenna}

Design and Simulation of an array patch antenna at $2.45 \mathrm{GHz}$. The main idea in this step is together two identical elements of designing the patch antenna in order to increase the gain and the directivity of the desired antenna performances. Figure. 3 shows the proposed structure of the used design. 


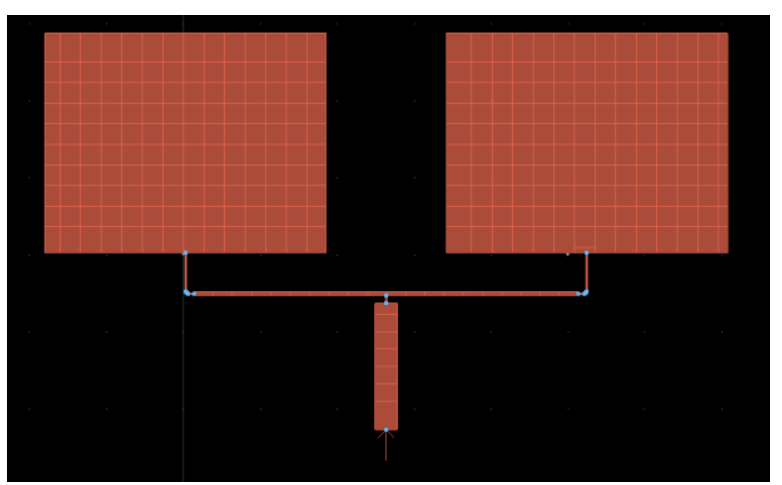

Figure.3 Layout of the array microstrip patch antenna

The important parameter to be considered is the maximum transfer of power, return loss of the designed antennas obtained from ADS Agilent, Where S11 represents the amount of energy sent to the amount of energy reflected Therefore, a graph of S11 of an antenna versus frequency is called its input reflection coefficient curve. it is shown in figure. 4 for single patch antenna and figure. 5 for array patch antenna that it has an impedance bandwidth for return loss less than $-10 \mathrm{~dB}$ around at $2.45 \mathrm{GHz}$.

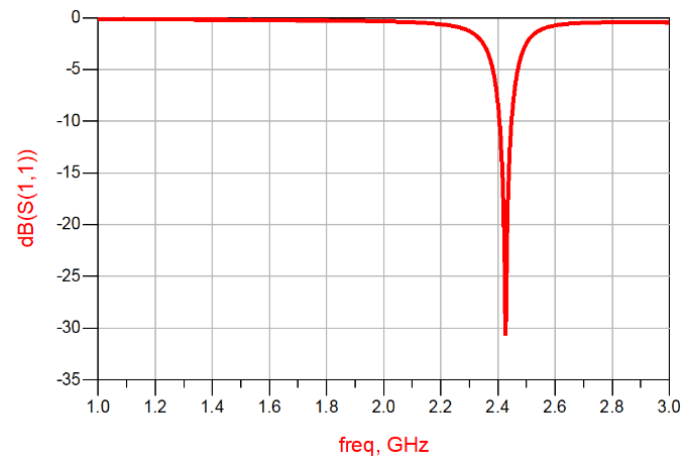

Fig.4. $\mathrm{S} 11$ of the proposed patch antenna resonating at $2.45 \mathrm{GHz}$

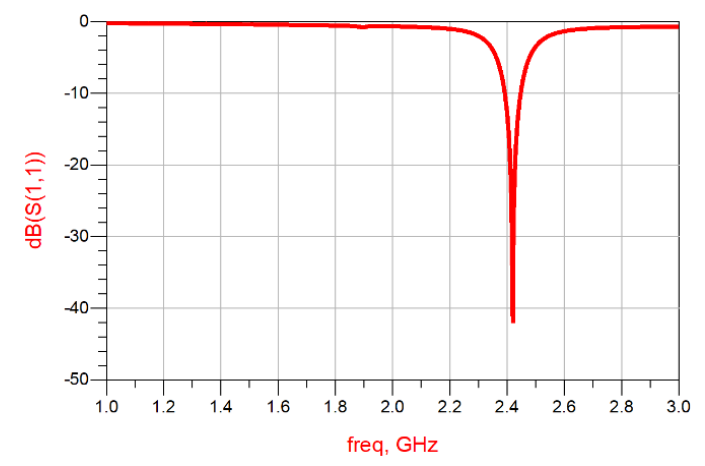

Fig.5. S11 of the proposed array patch antenna resonating at $2.45 \mathrm{GHz}$ 
The performance comparison between the designed single patch antenna and the array patch antenna is shown in Table 2. the table is shown simulation results for Return Loss (S11) values, VSWR, 3D directivity diagrams.

Table. 2: Comparison of microstrip patch antennas

\begin{tabular}{|c|c|c|}
\hline Performances & Single patch antenna & Array patch antenna \\
\hline S11 (dB) & -31.63 & -42 \\
\hline BW (MHz) & 128 & 170 \\
\hline Gain (dB) & 3.24 & 5.75 \\
\hline $\begin{array}{c}\text { Directivity } \\
\text { (dB) }\end{array}$ & 6.62 & 7.82 \\
\hline $\begin{array}{c}\text { Radiation } \\
\text { Efficiency } \\
(\%)\end{array}$ & 49 & 62 \\
\hline
\end{tabular}

From the analysis of this table, it can be seen that a designed antenna based on two elements provides good electrical performance. The work carried out in this project is in the field of the design, modeling, and simulation of printed antenna arrays for an important telecommunication application. The antenna network model to be simulated can be very useful for RFID applications.

\section{Active Patch Antenna}

The low noise amplifier LNA is one of the basic building blocks of a communication system responsible for shaping very weak signals from an antenna. It is usually located at the entrance of the receiver, to minimize line losses; therefore, it receives the signals directly from the antenna. The main function of a Low Noise Amplifier (LNA) is to amplify the signal while adding as little noise as possible. Since most commercial LNA circuits are often unstable and not matched to a standard impedance (such as 50), stabilization and matching networks are needed. In this case, the most critical requirement is the noise figure, which must be $<2.1 \mathrm{~dB}$. The main objective is to design an amplifier with the simplest possible architecture, which should operate in the microwave band. 
The circuit is a low noise amplifier (LNA) manufactured in InGaAs technology. The transistor used is the cf_ctk_CF00302_19930609. The two main parameters that must be taken into consideration are noise and gain. The simplicity of the LNA schematic should not make us forget that its realization is not dissociable from that of its constituent elements. The most suitable structure for a given need must therefore be sought, in terms of performance, surface area, and reproducibility. The analysis is done on a (2-3) GHz frequency range. According to the figure. $6, \mathrm{k}=1.583$ the determinant of the matrix $\mathrm{S}$ being $\Delta=0.248$; We have thus the factor $\mathrm{K}$ is greater than $1(\mathrm{~K}>1)$ and $\Delta<1$ so we are in the case where the transistor is unconditionally stable. Then it is possible to simultaneously adapt the input and output of the quadrupole.

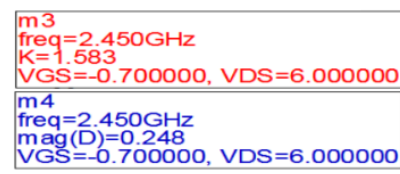

Eqn $\mathrm{D}=\left(\mathrm{S}(1,1)^{\star} \mathrm{S}(2,2)\right)-\left(\mathrm{S}(1,2)^{\star} \mathrm{S}(2,1)\right)$

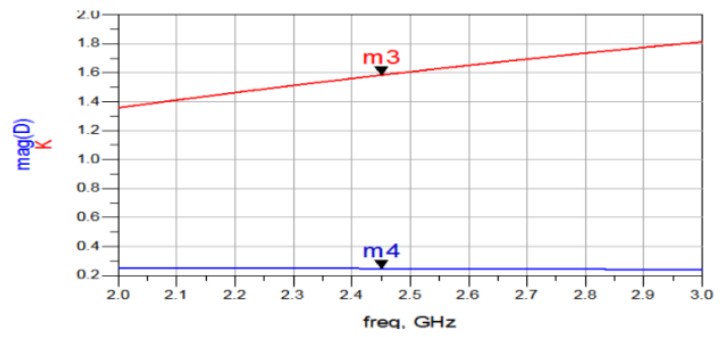

Figure 6. Simulated transistor stabilization response.

The matching network must adapt the input and output impedance of the transistor to the reference impedance of the measurement $(50 \Omega)$ for this we add the matching circuits in input and output. when be Integrating the obtained circuits to our configuration, we obtain a low noise amplifier circuit adapted to its input and output in figure.7.

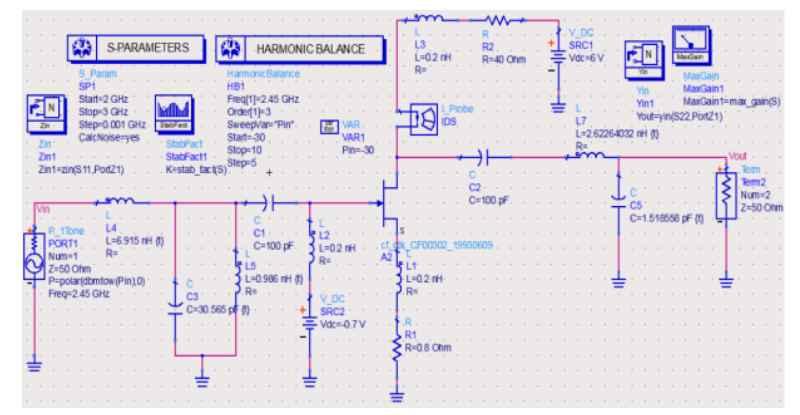

Figure 7. low noise amplifier circuit 
After completion design of a low noise amplifier in the microwave band at an operating frequency of $2.45 \mathrm{GHz}$, and determine the operating point of our transistor. The results allow obtained us to make an inference on the chosen transistor. The proposed design must provide a compromise between low power consumption, a minimum noise factor, and a high gain. In a second time, we proceeded to the adaptation of our low noise amplified by the addition of adaptation circuits at the input and at the output. From figure (8-9), We obtained a low noise figure around 0.42 and a gain of 37.246.

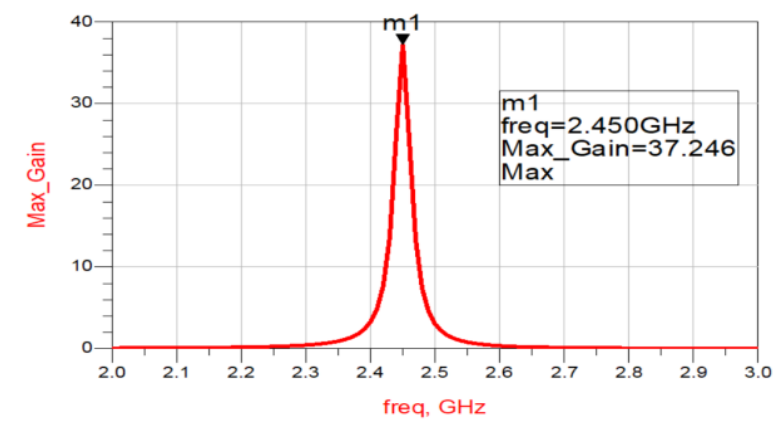

Figure.8 Simulated maximum gain parameter in LNA.

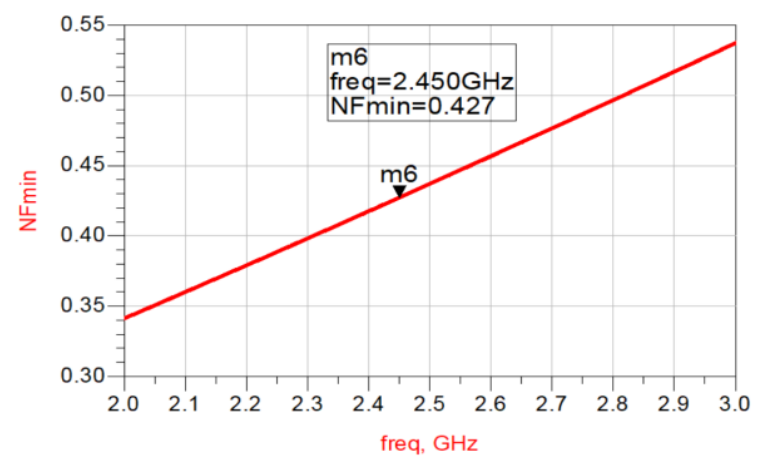

Figure.9 Simulated a low noise in LNA.

From figure.10, the curve of variation of the input and output signals of the amplifier, we can see that the output signal of the amplifier is sinusoidal and well amplified compared to its input signal. 


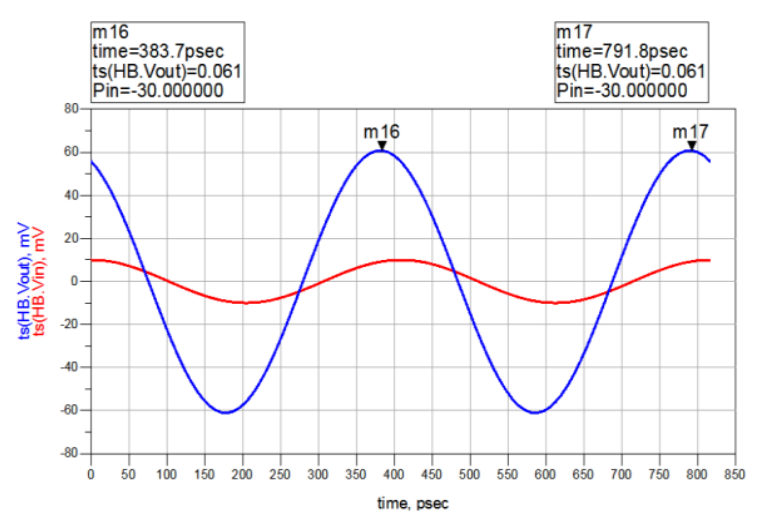

Figure.10 Comparison of the output and input signals of the amplifier in the time domain.

\section{Active integrated antennas}

The Active Integrated Antenna (AIA) design is shown in figure.11. The active integrated antenna consists of two parts. Part one is dealing with the active element while part two is dealing with the passive elements

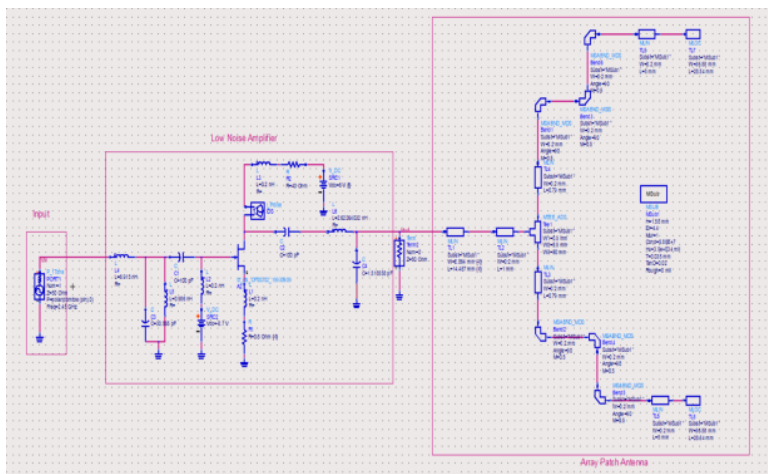

Figure.11 Active Integrated Antenna System

The active integrated antenna has been designed at the frequency of $2.45 \mathrm{GHz}$. In the proposed antenna, we have followed the mathematical approach of the transmission line model (TLM) where the important parameters are the resonant frequency (f0), the dielectric constant (cr) and the height of the substrate (h). As a result, the designed antenna (AIA) consists of:

- Array Patch Antenna based on Microstrip Technology

- Low Noise Amplifier based on AsGa Mosfet Transistor 
Academic Journal of Research and Scientific Publishing | Vol 3 | Issue 30

Publication Date: 5-10-2021

From the analysis of the obtained simulation results, we are able to determine the reflection and transmission coefficients of the active integrated antenna. Table. 3 resumes all obtained performance for the designed AIA system.

Table. 3 Performance summary of the designed AIA system

\begin{tabular}{|c|c|}
\hline Parameters & Values \\
\hline S11 $(d B)$ & -52.076 \\
\hline S22 $(d B)$ & -42.729 \\
\hline S12 $(d B)$ & -24.753 \\
\hline S21 $(d B)$ & 15.64 \\
\hline Gain & 36.682 \\
\hline NFmin & 0.430 \\
\hline
\end{tabular}

\section{Improvement the Gain of Active Integrated Antenna System}

In order to increase the obtained gain and increase its value, will add some enhancement elements (parasite elements) in the designed AIA system as the integration of the parasitic capacitor exactly in the LNA part (active antenna) for increasing the gain. One of the important parasitic elements for improving the gain of the designed circuit is the inductor element. We can also change the value of VDS applied voltage in drain for increasing the gain.

Table. 4 Performance summary after add some enhancement elements in the designed AIA system

\begin{tabular}{|c|c|}
\hline Parameters & Values \\
\hline S11 $(\mathrm{dB})$ & -30.58 \\
\hline S22 $(\mathrm{dB})$ & -21.39 \\
\hline $\mathrm{S} 12(\mathrm{~dB})$ & -24.35 \\
\hline
\end{tabular}


Academic Journal of Research and Scientific Publishing | Vol 3 | Issue 30

Publication Date: 5-10-2021

ISSN: 2706-6495

\begin{tabular}{|c|c|}
\hline S21 $(\mathrm{dB})$ & 16.69 \\
\hline Gain & 46.71 \\
\hline NFmin & 0.37 \\
\hline
\end{tabular}

Regarding table. 4 it can be seen that all used parasitic elements increases the gain; we obtained an excellent gain around 46 with low noise factor $(0.37)$.

\section{Circular polarization using defected patch structure (slot)}

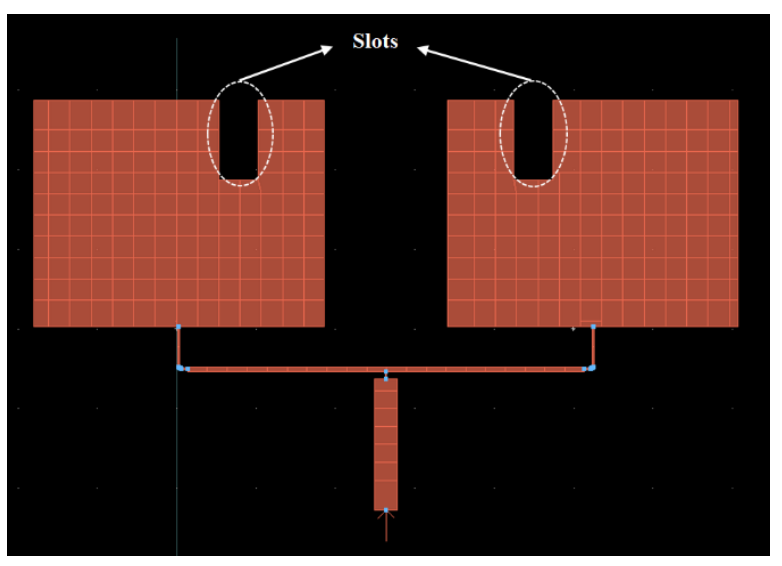

Figure.12 Layout of slots integration in the array patch antenna structure

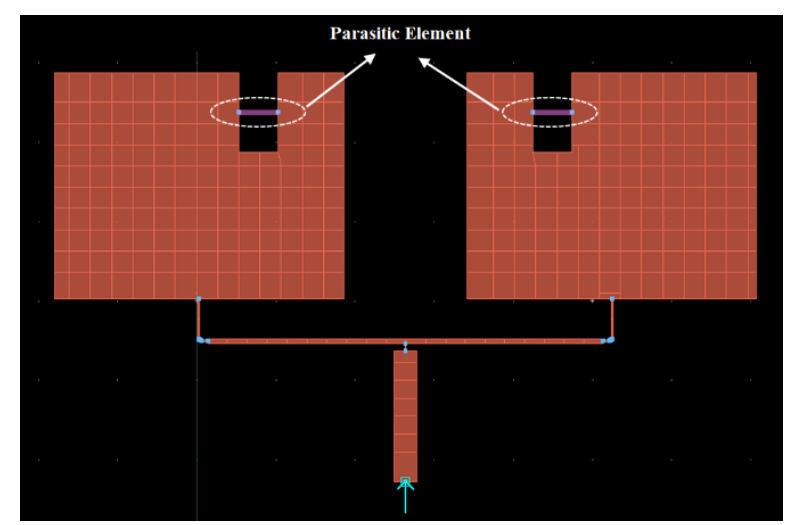

Figure.13 Layout of parasitic elements with slots integration in the array patch antenna structure 
One method that can use to improve the gain of the desired active integrated antenna (AIA) is circular polarization. Figure.12 presents the slots integration in the array patch antenna structure. These slots will be perturbing the current for increasing the gain. The next step is to integrate the parasitic elements (capacitor) in the design slots as shown the figure.13.

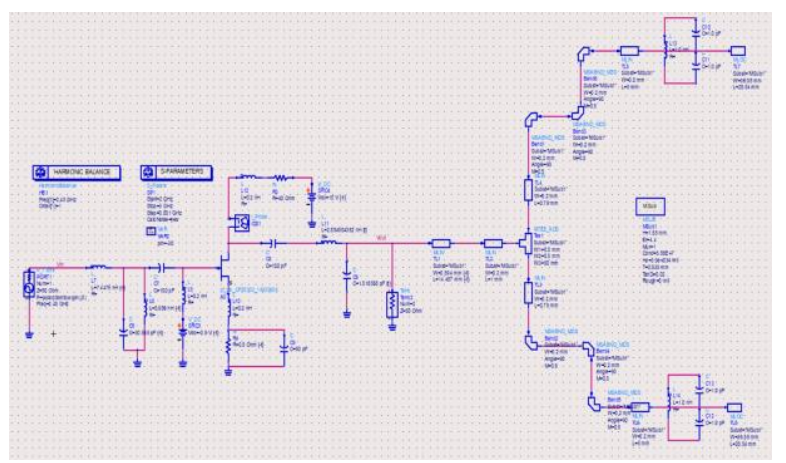

Figure.14 Final designed of AIA system

Figure.14 present the final designed active integrated antenna system after adding circular polarization and parasitic elements in the array microstrip patch antenna. From the analysis of the results in table. 5 it can be seen that the gain increase to 47 and the reflection parameter $\mathrm{S} 22$ increase to $-46.58 \mathrm{~dB}$. This is why the circular polarization and the parasitic elements have allowed us to obtain the desired performances.

Table. 5 Performance summary final of AIA system

\begin{tabular}{|c|c|}
\hline Parameters & Values \\
\hline S11 $(\mathrm{dB})$ & -25.79 \\
\hline S22 $(\mathrm{dB})$ & -46.58 \\
\hline S12 $(\mathrm{dB})$ & -24.32 \\
\hline S21 $(\mathrm{dB})$ & 16.721 \\
\hline Gain & 47.0 \\
\hline NFmin & 0.37 \\
\hline
\end{tabular}


The adaptation of the system for validate the results obtained in terms of the input and output impedance. From Analysis of these results, we observed the input and output impedance are fixed in $50 \mathrm{Ohm}$. Which shows that the designed system is well adapted.

For a radio (transmitter or receiver) to deliver power to an antenna, the impedance of the radio and transmission line must be well matched to the antenna's impedance. The parameter VSWR is a measure that numerically describes how well the antenna is impedance matched to the radio or transmission line it is connected to.

From analysis of the obtained results about the VSWR parameter, we have a good value in transmitting and receiving the data. This parameter must be $(1<\mathrm{VSWR}<2)$.

- In transmission of data: VSWR=1.06

- In receiving of data: VSWR=1.186

The Group Delay variations versus frequency is an essential factor which can cause distortion and degradation in the data, which are used for transmitting and receiving the data with low delay possible.

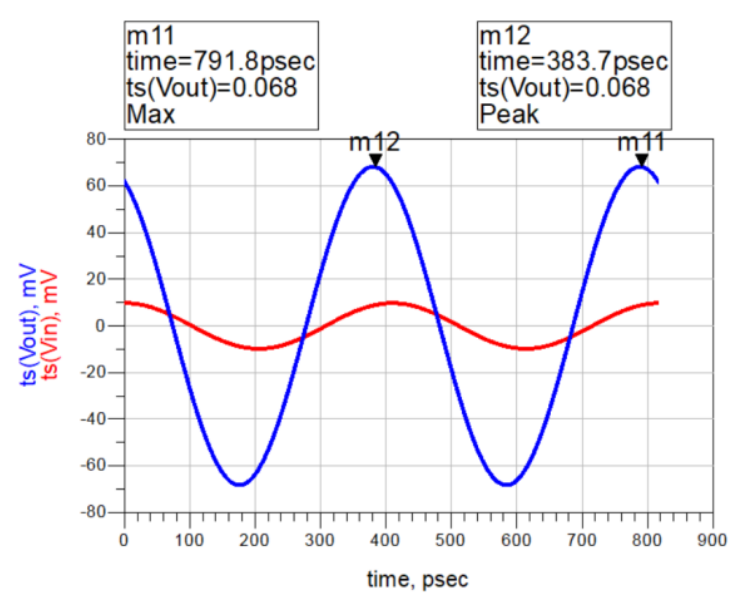

Figure.15 Comparison of the output and input signals of final of AIA system in the time domain

The figure. 15 presents the signals at the input and output of the designed AIA system in the time domain. 
From the curve of variation of the input and output signals of the Active Integrated Antenna we can see that the output signal of the AIA has the same frequency compared to its input signal.

\section{Conclusion}

In this paper, a novel Active Integrated Antenna AIA was proposed for the communication applications at microwave band from $2-3 \mathrm{GHz}$ frequency range to get high gain microstrip active antenna utilizes as transmitter and receiver as the same time and focused on the design based on a small size at a resonant frequency of $2.45 \mathrm{GHz}$. To prove the concept, firstly presented the design of the passive patch antenna that contains two parts based on microstrip technology for obtaining a high gain and directivity of the desired antenna. In the second step, the low noise amplifier LNA is designed to amplify the signal while adding as little noise as possible with the simplest possible architecture. after completing the design of a low noise amplifier and determine the operating point of our transistor at an operating frequency of $2.45 \mathrm{GHz}$, the results were obtained to make an inference on the chosen transistor a compromise between low power consumption, a minimum noise factor, and a high gain. We obtained a low noise figure around 0.42 and a gain of 37.246. In the next step, we combined the passive antenna with the active antenna to get an active integration antenna AIA. for Improvement the gain of the active integrated antenna system, we increased the obtained gain and increase its value by adding enhancement elements (parasite elements) in the designed AIA system as the integration of the parasitic capacitor in the AIA circuit for increasing the gain. after applying circulation polarization (CP) we obtained an excellent gain of around 47 with a low noise factor (0.37).

\section{Acknowledgment}

Co-Prof. Mohammad Nasser Ajour is grateful for the support provided during writing a master's thesis 
Academic Journal of Research and Scientific Publishing | Vol 3 | Issue 30

Publication Date: 5-10-2021

\section{Reference}

Garg et al. (2001), Microsrrip Antenna Design Handbook, Artech House.

Khoshniat et al. (2011), A linearly polarized active integrated square microstrip patch antenna, Antennas and Propagation (APSURSI), pp.3082-3084. IEEE.

Rogier, Declercq (2010), Active Integrated Wearable Textile Antenna With Optimized Noise Characteristics, Antennas and Propagation. pp.3050 -3054, IEEE.

Peter T. et al. (2007), Design of low noise amplifier with Active Integrated Antenna at 5 GHz”, Applied Electromagnetics, APACE 07, pp.1-5, IEEE.

T, Itoh (1997), Active Integrated Antennas for Wireless Applications", Microwave conference proceedings, APMC, vol.1, pp.309-312, AsiaPacific, IEEE.

Copyright @ 2021 Engr. AHMED. S. ALZAHRANI, Prof. ADNAN. M. AFFANDI, AJRSP. This is an open-access article distributed under the terms of the Creative Commons Attribution License (CC BY NC).

Doi: doi.org/10.52132/Ajrsp.e.2021.304 